\title{
Will DSM-5 change the way we practise psychiatry?
}

\author{
Varuni de Silva
}

\section{Summary}

The DSM-5 is expected to be released in May 2013. Much new knowledge about psychiatric disorders has emerged since then and it was thought that most of this would be incorporated into the new classification. The descriptive approach of the DSM IV has been retained in the DSM-5 and it still remains primarily a syndromal classification system. The DSM- 5 includes several new categories such as attenuated psychosis and mixed anxiety disorder in Section
III as disorders which need further study. The personality disorder category has undergone major changes including the provision for identification of personality traits. Diagnostic criteria of some older categories have been changed with the removal of the bereavement exclusion criteria for diagnosing depression leading to much discussion.

SL J Psychiatry 2012; 3 (1):1-2
The DSM-5 is expected to be released in May 2013 (1). This is the first full revision of the DSM since the DSM IV was published in 1994. Much new knowledge about psychiatric disorders has emerged since then and it was thought that most of this would be incorporated into the new classification. The DSM is one of two main classification systems used by psychiatrists worldwide. Initially with the DSM-5, a paradigmal shift in the system of classification was anticipated. A classification system based on aetiology or a dimensional system were possibilities. However an aetiological classification requires more knowledge on the pathogenesis of psychiatric disorders than what is currently known. Despite the advances in brain imaging, biomarkers and molecular genetics psychiatrists are still not able to clearly delineate the pathogenesis of psychiatric disorders. Therefore it seems that an aetiological classification system is many years away.

The DSM III diagnostic system adopted a descriptive approach in which disorders were characterized in terms of symptoms that could be elicited by patient report, direct observation, and measurement (2). This descriptive approach has been retained in the DSM- 5 and it still remains primarily a syndromal classification system. Some diagnostic criteria have changed but the most important proposed changes are the introduction of new disease categories some of which straddle the boundaries between 'normal' and 'pathological'.

The two proposed categories that sparked off the most debate were that of attenuated psychosis syndrome and mixed anxiety depressive disorder. The diagnostic criteria for attenuated psychosis syndrome requires either delusions/delusional ideas, or hallucinations/ perceptional abnormalities or disorganized speech/communication to be present in attenuated form with relatively intact reality testing, but of sufficient severity and/or frequency to warrant clinical attention and to be present at least once per week for the past month.

Although these criteria were proposed with a view to identifying individuals at risk of psychosis, one problem was that the criteria did not differentiate adequately between normality and psychosis. This would have led to an over diagnosis of individuals in this risk category leading to inappropriate treatment and labelling of people. Protest about the inclusion of this category has now led to the DSM-5 recommending this condition for further study in section III (3). Section III of the DSM-5 includes conditions that require further research (1). A similar decision was taken regarding the mixed anxiety depressive disorder category too.

The need to include categories such as attenuated psychosis and mixed anxiety depressive disorder are based as much on service needs as research findings. In some developed countries, ironically, access to treatment is dependent on medical insurance and medical insurance does not provide coverage unless the patient has a 'legitimate' diagnosis. In less resourced middle income countries like Sri Lanka, free health care ensures that patients can access psychiatric services regardless of whether their distress can be allocated a diagnosis or not.

Several other new diagnostic categories have been added which reflect recent advances in understanding psychiatric illness. With increasing evidence of the existence of mild cognitive impairment, its association with Alzheimer's disease and with the availability of screening instruments which detect it, efforts are made to identify those with this condition $(4,5)$. The new category, neurocognitive disorder includes three broad syndromes: Delirium, Major Neurocognitive Disorder, and Mild Neurocognitive Disorder (1). Minor Neurocognitive Disorder corresponds closely to the clinical entity of mild cognitive impairment.

The DSM IV criteria for diagnosis of major depressive disorder includes a footnote for differentiating between bereavement and depressive disorder. A diagnosis of depressive disorder is not made unless the symptoms of depression following bereavement have lasted more than two months. This bereavement exclusion for diagnosis of depression has been removed from the DSM-5. This has been supported by evidence that bereavement related depression within the first two months after the death of a loved one resembles non-bereavement related depression (6). It is also interesting to note that the ICD-10 does not recognize this exclusion. Again this modification has resulted in concerns that this could lead to 'medicalization' of bereavement.

A new disorder, 'Persistent complex bereavement related disorder' has been added to Section III. The criteria suggest a persistent bereavement state in individuals experiencing some features of bereavement after twelve months following the death of a close family member or close friend. This again is in response to evidence suggesting the existence of 'prolonged grief' or 'traumatic grief'. 
The section on substance use disorders has been expanded to accommodate 'addiction disorders'. Internet use disorder and caffeine use disorder are new categories included in Section III of this category. This is the first time 'behavioural addictions' have been recognised as a category although DSM IV included gambling disorder as an Impulse Control Disorder. Gambling disorder and internet use disorder can be termed 'behavioural addictions', the latter arising as a result of recent social changes. It will be interesting to see which other behavioural addictions may in the future be proposed for inclusion under this category.

The category for personality disorders has undergone substantial changes and clinicians may feel most unfamiliar with this category. DSM -5 describes the personality disorder category as a hybrid dimensional-categorical model for personality and personality disorder (1). Only six specific personality disorder types have been retained from the 10 in DSM IV. The definition of personality disorder too has changed. DSM-5 states 'The essential features of a personality disorder are impairments in personality (self and interpersonal) functioning and the presence of pathological personality traits'. A new 'Levels of personality functioning scale' is included for assessment of impairment in personality functioning. The most striking change though is the introduction of five personality trait domains: negative affectivity, detachment, antagonism, disinhibition vs. compulsivity, and psychoticism. The assessment process required for identifying personality types and traits is complex and commentaries have indicated many concerns and discomfort with the changes (7).

Many other categories have undergone change. One example is the new category Neurodevelopmental disorders which now contains the diagnosis autism spectrum disorder. This diagnosis includes autistic disorder (autism), Asperger's disorder, childhood disintegrative disorder, and pervasive developmental disorder not otherwise specified. With the introduction of new categories and changes in the diagnostic criteria of previous categories one major concern is that prevalence of some disorders may increase dramatically with the use of new criteria. Over diagnosis can also lead to unnecessary treatment.

The publication of the DSM-5 will also significantly influence the ICD-11 expected to be published in 2014. While some scientists have argued for integrating the two system this seem unlikely at present (8).

The strength of the DSM as well as the ICD systems of classification is high reliability. However the same cannot be said about the validity of many of the diagnostic categories. Eventually the DSM- 5 will be judged by clinicians for its ease of use, its ability to meet service needs and how well it serves the needs of service users. In addition to its utility in clinical practise settings, critical analysis and feedback regarding the validity and reliability of syndromes and its validity in research settings will be evaluated as an on-going process.

\section{Declaration of interest \\ None}

\section{Varuni de Silva}

Senior Lecturer in Psychiatry,

Faculty of Medicine, University of Colombo, Sri Lanka

E mail: varunidesilva2@yahoo.co.uk

\section{References}

1. DSM-5: The Future of Psychiatric Diagnosis http://www. dsm5.org/Pages/Default.aspx

2. Ed. Kuppfer DJ,First MB,Regier DAA Research agenda for DSM-V, Washington, D.C American Psychiatric Association 2002

3. Strakowsk SM, Frances AJ, What's Wrong With DSM-5? http://www.medscape.com/viewarticle/763886

4. Nasreddine ZS, Phillips NA, Bedirian V, et al. The Montreal Cognitive Assessment, MoCA: a brief screening tool for mild cognitive impairment. Journal of the American Geriatrics Society 2005; 53: 695-9.

5. Karunaratne S, Hanwella R, de Silva V. Validation of the Sinhala version of the Montreal Cognitive Assessment in screening for dementia. Ceylon Med J. 2011 Dec;56(4):14753

6. Zisook S, Shear K, Kendler K.. Validity of the bereavement exclusion criterion for the diagnosis of major depressive episode. World Psychiatry. 2007; 6(2): 102-107

7. Pilkonis PA, Hallquist MN, Morse JQ, Stepp SD Striking the (Im)Proper Balance between Scientific Advances and Clinical Utility: Commentary on the DSM-5 Proposal for Personality Disorders. Personal Disord. 2011 January 1; 2(1): 68-82.

8. Jablensky A. Towards ICD-11 and DSM-V: issues beyond 'harmonisation'. Br J Psychiatry. 2009 Nov;195(5):379-81. 